\section{Antibody drug discovery and development}

Abbott Laboratories

Abgenix

Ablynx

Agensys

Affimed Therapeutics

Alexion Antibody Technologies

Amgen

Biogen idec

Biolnvent

Cambridge Antibody Technology

Centocor

Domantis

Dyax

Genentech

Iclectus

Drug discovery and development; Humira therapeutic monoclonal antibody Antibody drug discovery and development via proprietary XenoMouse and XenoMax technologies for producing fully human monoclonal antibodies

Development of natural single-domain antibodies (nanobodies)

Antibody drug discovery

Antibody drug discovery and development; phage-display human Fab libraries

Drug discovery and development; Enbrel therapeutic monoclonal antibody

Drug discovery and development; therapeutic monoclonal antibodies

Rituxan and Zevalin

Antibody development in proprietary n-CoDeR phage-display human scFv library Phage-display human scFv libraries; antibody drug discovery and development Antibody and protein drug discovery and development; Remicade and ReoPro therapeutic monoclonal antibodies

Development of domain antibodies as therapeutics and for target validation Drug discovery and development; phage-display human Fab libraries Antibody drug discovery and development; Xolair, Herceptin and Raptiva therapeutic monoclonal antibodies

Development of intracellular antibodies (intrabodies) as therapeutics and diagnostics

\section{ILEX Oncology}

ImClone

Medarex

Drug discovery and development; CAMPATH monoclonal therapeutic antibody

Antibody drug discovery and development; Erbitux therapeutic monoclonal antibody

UltiMAb package comprising HuMAb-Mouse and other similar mice for producing

fully human antibodies

MorphoSys

Ortho Biotech

Protein Design Labs

Regeneron

Phage-display library of synthetic antibodies; antibody drug development

Antibody drug discovery; Orthclone OKT3 monoclonal therapeutic antibody

Antibody drug discovery; Zenapax monoclonal therapeutic antibody

Development of hybrid antibody C-region-receptor molecules (Traps) as therapeutics

Xerion Pharmaceuticals

Xoma

XCalibur proprietary antibody-based target-inhibition technology

Antibody drug discovery and development; Raptiva monoclonal therapeutic antibody
Phage-display human antibody libraries

Abbott Park, llinois

Fremont, California

Zwijnaarde, Belgium

Santa Monica, California

Heidelberg, Germany

San Diego, California

Thousand Oaks, California

Cambridge, Massachusetts

Lund, Sweden

Cambridge, UK

Malvern, Pennsylvania

Cambridge, UK

Cambridge, Massachusetts

London, UK

San Antonio, Texas

New York, New York

Princeton, New Jersey

Martinsried, Germany

Bridgewater, New Jersey

Mountain View, California

Tarrytown, New York

Martinsried, Germany

Berkeley, California

Cambridge, UK

San Diego, California

Antibody suppliers

liers and services

AbCam

Polyclonal and monoclonal antibodies; customized antibody development;

off-the-shelf and custom proteins and peptides; protein-expression services

Affinity BioReagents

Monoclonal and polyclonal antibodies

Affitech Phage-display human antibody libraries, screening, scFv and Fab

production, regeneration to full monoclonal antibodies

AnaSpec

Off-the-shelf and customized antibodies and peptides

Antibodies by Design

Custom monoclonal antibodies made from the proprietary Morphosys HuCAL

GOLD library

Antibodies Incorporated

Catalogue and custom antibodies; reagents and kits for immunochemistry and immunoassay

Aves Labs

BD Biosciences Pharmingen

Catalogue and custom polyclonal chicken antibodies

Biogenes

Biogenesis

Catalogue and custom antibodies; kits for immunoassays

Custom monoclonal and polyclonal antibody and immunoassy development

Antibodies and immunoassay-related reagents; bulk polyclonal and monoclonal antibodies; custom antibody development and conjugation service

Biomeda

BioProtein Technologies

ELISA kits, monoclonal and polyclonal antibodies

Human-antibody production in transgenic rabbits

Custom peptides; catalogue and custom antibodies

Catalogue antibodies; custom antibody production service

Biotrend

Peptide synthesis and modification, antibody production, ELISA

Cambridge Research Biochemicals

Chemicon

Cytomyx

Delta Biolabs

EMD Biosciences

Eurogentec

Fusion Antibodies

Gallus immunotech

Genetel

Genovac

GenWay Biotech

GTC Biotherapeutics

Harlan Sera-Lab
Catalogue mono- and polyclonal antibodies; equipment and reagents for immunology; approved antibody-based in vitro diagnostic kits Antibodies for kinases, receptors and ion channels Catalogue antibodies

Antibodies and other immunology tools for life-sciences research Catalogue and custom antibodies; custom antibody-based protein arrays Custom polyclonal, monoclonal, Fab or scFv production

Chicken polyclonal antibodies

Antibodies for proteomics; chicken antibody specialists; antibody services

Functional antibodies against G-protein-coupled receptors

Primary and secondary chicken antibodies

Human-antibody production in transgenic goats

Custom monoclonal and polyclonal antibody production, peptide synthesis,

purification, conjugation and hybridoma development
Golden, Colorado

Oslo, Norway

San Jose, California

Martinsried, Germany

Davis, California

Tigard, Oregon

San Diego, California

Berlin, Germany

Poole, UK

\section{\begin{tabular}{l} 
Fost \\
Paris \\
Cam \\
Colo \\
Billin \\
Tern \\
\hline
\end{tabular}}

Cambridge, UK

San Diego, California

Seraing, Belgium

Belfast, UK

Wildwood, MO

Madison, Wisconsin

Freiburg, Germany

San Diego, California

Framingham, Massachussetts

Loughborough, UK abbottcom

www.abgenix.com

www.ablynx.com

www.agensys.com

www.affimed.com

www.alxnsd.com

www.amgen.com

www.biogen.com

www.bioinvent.com

www.cambridgeantibody.com

www.centocor.com

www.diversys.co.uk

www.dyax.com

www.iclectus.com

www.ilexonc.com

www.imclone.com

www.medarex.com

www.morphosys.com

www.orthobiotech.com

www.pdl.com

www.regeneron.com

www.xerion-pharma.com

www.xoma.com www.abcam.com

www.abgent.com

www.bioreagents.com

www.affitech.com

www.anaspec.com

www.antibodyservices.com

www.antibodiesinc.com

www.aveslab.com

www.bdbiosciences.com/pharmingen www.biogenes.de

www.biogenesis.co.uk

biomeda.com

www.bioprotein.com

www.biosource.com

www.biotrend.com

www.crb.gb.com

www.chemicon.com

www.cytomyx.com

www.deltabiolabs.com

www.emdbiosciences.com

www.eurogentec.be

www.fusionantibodies.com

www.gallusimmunotech.com

www.genetel-lab.com

www.genovac.com

www.genwaybio.com

www.gtc-bio.com

www.harlanseralab.co.uk 


$\begin{array}{llll}\begin{array}{lll}\text { Company } \\ \text { Imgenex }\end{array} & \text { Products/activity } & \text { Location } & \text { URL } \\ \text { Innovative Research } & \text { Catalogue and custom polyclonal and monoclonal antibodies; ELISA kits } & \text { San Diego, California } & \text { www.imgenex.com } \\ \text { Lonza Biologics } & \text { Custom and catalogue antibodies } & \text { Southfield, Michigan } & \text { www.innov-research.com } \\ & \begin{array}{l}\text { Contract manufacture of therapeutic antibodies and recombinant proteins from } \\ \text { mammalian cell culture }\end{array} & \text { Portsmouth, New Hampshire } & \text { www.lonzabiologics.com } \\ \text { Mabtech } & \begin{array}{l}\text { Catalogue monoclonal antibodies for cytokine detection in ELISpot, ELISA and } \\ \text { intracellular staining; custom monoclonal production }\end{array} & \text { Nacka Strand, Sweden } & \text { www.mabtech.com } \\ \text { Novus Biologicals } & \text { Custom and catalogue antibodies } & \text { Littleton, Colorado } & \text { www.novus-biologicals } \\ \text { Open Biosystems } & \text { Antibody services } & \text { Huntsville, Alabama } & \text { www.openbiosystems.com } \\ \text { PeproTech } & \text { Anti-cytokine antibodies } & \text { Rocky Hill, New Jersey } & \text { www.peprotech.com } \\ \text { Planet Biotechnology } & \text { Production of recombinant proteins in genetically modified plants } & \text { Hayward, California } & \text { www.planetbiotechnology.com } \\ \text { Progen Biotechnik } & \text { Catalogue antibodies; in vitro diagnostic assays } & \text { Heidelberg, Germany } & \text { www.progen.com } \\ \text { Research Diagnostics } & \text { Antibody distributors } & \text { Flanders, New Jersey } & \text { www.researchd.com } \\ \text { Serotec } & \text { Custom and catalogue antibodies } & \text { Raleigh, North Carolina } & \text { www.serotec.com } \\ \text { Sigma-Aldrich } & \text { Catalogue monoclonal and polyclonal antibodies; biochemicals and reagents } & \text { St Louis, Missouri } & \text { www.sigmaaldrich.com } \\ \text { SouthernBiotech } & \text { Catalogue and custom polyclonal and monoclonal antibodies and reagents } & \text { Birmingham, Alabama } & \text { www.southernbiotech.com } \\ \text { Virusys Corporation } & \text { for immunological research } & \text { Sykesville, Maryland } & \text { www.virusys.com }\end{array}$

\section{Immunoassay and immunochemistry}

Active Motif

Alpha Diagnostics

Amersham Biosciences

Assay Designs

BD Biosciences Immunocytometry

Systems

Beecher Instruments

Bender MedSystems

Bio-Rad
Biosite

GenBio

InnoGenex

Invitrogen

Jerini Array Technology

\section{Novagen}

Panomics

PerkinElmer Life Sciences

Pierce Biotechnology

\section{Prionics}

ProSci

QED Bioscience

R\&D Systems

Roche Applied Science

Thermo Shandon

Upstate

Vision Biosystems

Zymed

Zyomyx

\section{ELISA kits for transcription-factor assays; antibodies; peptide nucleic acids for gene silencing; kits and reagents for cell fractionation; nucleic-acid isolation Custom peptides and polyclonal antibodies; kits for ELISA and western blotting Reagents for immunochemistry and immunoassays \\ MAP kinase immunoassays (ELISA), Antibody Shop antibodies \\ FACS range of flow cytometers}

\section{Automated and manual tissue microarray instruments and accessory} tissue-arrayer products

ELISA systems for research and in vitro diagnosis; instant ELISAs for cytokines and other immune-system molecules in humans and other species Products, instruments and software for life-sciences research; Bio-Plex system for multiplexing antibody-type assays; EU-approved test for BSE Diagnostic antibodies; proprietary Omniclonal phage-display system for human antibody library development

ImmunoDOT and ImmunoWELL assays for clinical use; assays for antibodies characteristic of Lyme disease, autoimmunity, mononucleosis

Breast cancer tissue arrays; contract services for gene-expression profiling, drug-target localization and validation

Antibodies, fluorescent-tagged antibodies; immunohistochemistry kits Substrate identification service for kinases using pepSTAR peptidemicroarray technology Antibody array-based cytokine assay kit TranSignal human cytokine antibody array Automated immunoassay systems

Components and consumables for automated systems in molecular biology, protein chemistry, sample handling, immunology and chromatography EU-approved BSE tests

Catalogue and custom antibodies; immunoassays

Catalogue and custom antibodies; immunoassay kits

Cytokines; antibodies; ELISA kits; flow cytometry kits Reagents and kits for molecular biology, functional genomics and proteomics Antibodies, immunohistochemical reagents, general laboratory equipment Catalogue polyclonal and monoclonal antibodies; immunoprecipitation kits BOND immunohistochemistry equipment and reagents Antibodies, ELISA kits, ISH kits and immunohistopathology Human-cytokine profiling biochip system

\section{Carlsbad, California $\quad$ www.activemotif.com}

San Antonio, Texas www.4adi.com Uppsala, Sweden Ann Arbor, Michigan

San Jose, California www5.amershambiosciences.com www.assaydesigns.com www.bdbiosciences.com

\begin{tabular}{ll} 
Sun Prairie, Wisconsin & www.beecherinstruments.com \\
Vienna, Austria & www.bendermedsystems.com \\
Hercules, California & www.bio-rad.com \\
San Diego, California & www.biosite.com \\
San Diego, California & www.genbio.com \\
San Ramon, California & www.innogenex.com \\
\hline Carlsbad, California & www.invitrogen.com \\
Berlin, Germany & www.jerini.com
\end{tabular}

Berlin, Germany

Madison, Wisconsin
Redwood City, California
Boston, Massachusetts
Rockford, Illinois
Schlieren, Switzerland
Poway, California
San Diego, California
Minneapolis, Minnesota
Lewes, UK
Runcorn, UK
Charlottesville, Virginia
Mount Waverley, Australia
South San Francisco, California
Hayward, California

www.novagen.com www.panomics.com lifesciences.perkinelmer.com www.piercenet.com

www.prionics.ch www.prosci-inc.com www.qedbio.com www.RnDSystems.com www.roche-applied-science.com www.thermo.com/eThermo www.upstate.com www.vision-bio.com www.zymed.com www.zyomyx.com

\section{General}

Calbiochem

Cambio

Carl Zeiss

Chroma Technology

Dynal

Molecular Probes

MP Biomedicals

Peptide Specialty Labs

USB

see advertisement
Biochemicals and immunochemicals for life-science research STAR FISH chromosome paints; suppliers of specialized biochemicals Imaging systems; automated systems for ultra-high throughput screening Filters for UV, visible and infra-red microscopy

Magnetic-bead based separation technology Fluorescence-based detection reagents and systems Reagents and biochemicals for life-sciences research Custom peptides. Biochemicals and reagents for molecular biology
San Diego, California Cambridge, UK Jena, Germany Rockingham,Vermont Oslo, Norway Eugene, Oregon Aurora, Ohio Heidelberg, Germany Cleveland, Ohio www.calbiochem.com www.cambio.co.uk www.zeiss.de www.chroma.com www.dynal.no www.probes.com www.icnbiomed.com www.pslgmbh.com www.usbweb.com 